\title{
Extension of the Williams-Clapper model to stacked nondiffusing colored coatings with different refractive indices
}

\author{
Lionel Simonot \\ Laboratoire de Métallurgie Physique, UMR CNRS 6630, Boulevard Marie et Pierre Curie, BP 179, \\ 86962 Futuroscope Chasseneuil Cedex, France
}

Mathieu Hébert and Roger D. Hersch

Ecole Polytechnique Fédérale de Lausanne (EPFL), Switzerland, School of Computer and Communication Sciences, INF/Station 14, CH-1015 Lausanne, Switzerland

\begin{abstract}
Received September 9, 2005; accepted December 8, 2005; posted January 10, 2006 (Doc. ID 64666)
We propose a model for predicting the reflectance and transmittance of multiple stacked nonscattering coloring layers that have different refractive indices. The model relies on the modeling of the reflectance and transmittance of a bounded coloring layer, i.e., a coloring layer and its two interfaces with neighboring media of different refractive indices. This model is then applied to deduce the reflectance of stacked nonscattering layers of different refractive indices superposed with a reflecting diffusing background that has its own refractive index. The classical Williams-Clapper model becomes a special case of the proposed stacked layer model. (C) 2006 Optical Society of America

OCIS codes: $080.0080,080.2710,160.0160,350.2450$.
\end{abstract}

\section{INTRODUCTION}

Predicting the reflectance factor of multilayer samples such as printed or painted samples requires modeling of the interaction of light with a medium made of several superposed layers that have different spectral absorbances, light scattering properties, and possibly different refractive indices. Two classical models enable prediction of spectral reflectance factors: the Kubelka-Munk model, ${ }^{1}$ and the Williams-Clapper model. ${ }^{2}$ The Kubelka-Munk model and its extensions ${ }^{3,4}$ are restricted to diffusing layers all having the same index of refraction. The WilliamsClapper model is restricted to a single coloring nondiffusing layer superposed on top of a diffusing layer of the same refractive index.

In the present contribution, we propose a model predicting the reflectance of a diffusing support coated with nondiffusing coloring layers of different refractive indices. Fresnel reflections and refractions occur at the interfaces between neighboring layers. In each of the nondiffusing layers, pencils of light propagate along straight lines, are absorbed according to Beer's law, and are internally reflected or refracted into an adjacent layer in a direction given by Snell's laws and with a proportion given by the Fresnel formulas. Beneath the coloring layers, the diffusing support reflects toward the coloring layers a Lambertian light composed of an infinity of pencils of light that have uniformly distributed orientations. Neither the Kubelka-Munk model nor the Williams-Clapper model is able to predict the reflectance of such a multilayer sample.

The Kubelka-Munk model ${ }^{1}$ predicts the reflectance and transmittance of a diffusing and absorbing layer for light propagating according to the layer's normal direction. This model has been extended by Saunderson to account for the internal reflections at the interface between the scattering medium and the air, ${ }^{5}$ by Kubelka to account for diffuse light, ${ }^{3}$ and again by Kubelka ${ }^{4}$ to account for multiple superposed layers all having the same index of refraction.

The Williams-Clapper model, ${ }^{2}$ in contrast to the Kubelka-Munk model, requires the color layer to be nondiffusing. In the original model, the illuminating light is collimated and incident at an angle of $45^{\circ}$ and the exiting light is captured by a radiance detector at a zero angle. Extensions have been proposed for any measuring geometry. ${ }^{6-8}$ The Williams-Clapper model computes for each pencil of the diffuse light reflected by the reflecting diffuse substrate its absorption according to Beer's law and its reflectance at the interface with the air due to the Fresnel reflectivity. The attenuation of diffuse light due to absorption in the coloring layer and reflection at the interface with the air is thus obtained by summing up the attenuation of all light pencils composing the diffuse light. However, the Williams-Clapper model is limited to a color layer of the same refractive index as the underlying reflecting diffuse substrate.

Since the Kubelka-Munk model is not applicable to nonscattering layers, we pursue the Williams-Clapper approach in order to predict the reflectance of a diffusing support superposed with several nondiffusing coloring layers of different refractive indices. The resulting comprehensive multilayer reflectance model includes as special cases the Williams-Clapper model ${ }^{2}$ as well as the air-paint ${ }^{7}$ and the air-varnish-paint ${ }^{9}$ reflection models. 
In Section 2, we recall the basics of light reflection and transmission at an interface both for collimated and for diffuse natural light. In Section 3, we first establish the reflectance and transmittance of a single nonscattering coloring layer bounded by two interfaces. By replacing the reflectance (respectively, transmittance) of a simple interface between two media with the reflectance (respectively, transmittance) of a bounded layer, we are able to deduce the reflectance and transmittance of multiple stacked nonscattering layers of different refractive indices. In Section 4 , we compute the global reflectance and the bidirectional reflectance distribution function (BRDF) of stacked nonscattering layers superposed on top of a diffusing medium that has its own intrinsic reflectance and refractive index. The Williams-Clapper model becomes a special case of the proposed comprehensive multilayer reflectance prediction model. In Section 5, we draw the conclusions.

\section{BASIC CONCEPTS: REFLECTION, TRANSMISSION, AND ABSORPTION}

All irradiances, reflectances, transmittances, and absorption coefficients are wavelength dependent.

When a light pencil reaches an interface between two media $j$ and $k$ of different refractive indices $n_{j}$ and $n_{k}$, one part is reflected and one part is transmitted (refracted). The reflection and refraction directions satisfy Snell's laws (Fig. 1):

$$
n_{j} \sin \theta_{j}=n_{k} \sin \theta_{k} .
$$

For the considered interfaces, within the visible wavelength range, we assume that the imaginary part of the refractive index is very small compared with the real part. Nevertheless, all expressions can be generalized to complex refractive indices.

The reflectivity and the transmittivity of the interface, i.e., the fraction of incident irradiance that is reflected and transmitted by the interface, are given by the Fresnel formulas. They depend on the polarization of the incident light $^{10}$ and may be expressed in terms of the reflectivity and transmittivity associated with polarization in both the parallel and the perpendicular directions with respect to the incidence plane. The reflectivity for the parallel component, denoted by superscript $(p)$, is

$$
R_{j k}^{(p)}\left(\theta_{j}\right)=\frac{\tan ^{2}\left(\theta_{j}-\theta_{k}\right)}{\tan ^{2}\left(\theta_{j}+\theta_{k}\right)},
$$

and the reflectivity for the perpendicular component, denoted by superscript $(s)$, is

$$
R_{j k}^{(s)}\left(\theta_{j}\right)=\frac{\sin ^{2}\left(\theta_{j}-\theta_{k}\right)}{\sin ^{2}\left(\theta_{j}+\theta_{k}\right)}
$$

In this paper, we consider that the incident light is incoherent and unpolarized (natural light). The directions of vibration of natural light vary rapidly in a random manner. The wave component polarized parallel to the incidence plane, averaged over all the directions of vibration, forms an irradiance $W_{i}^{(p)}$. The average wave component polarized perpendicular to the plane of incidence

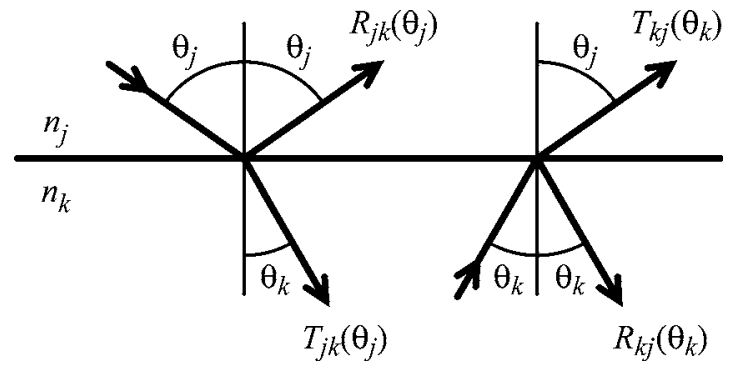

Fig. 1. Reflection and transmission of light at an interface between two media of refractive indices $n_{j}$ and $n_{k}$.

forms an irradiance $W_{i}^{(s)}$. Since natural light is incoherent and unpolarized, the two linearly polarized irradiance components are independent and equal:

$$
W_{i}^{(p)}=W_{i}^{(s)}=W_{i} / 2 .
$$

The total incident irradiance $W_{i}$ is the sum of the two linearly polarized irradiances:

$$
W_{i}=W_{i}^{(p)}+W_{i}^{(s)} .
$$

When the irradiance $W_{i}$ is reflected by the interface, the reflected irradiance $W_{r}$ is composed of a parallelly polarized component $W_{r}^{(p)}=R_{j k}^{(p)}\left(\theta_{j}\right) W_{i}^{(p)}$ and a perpendicularly polarized component $W_{r}^{(s)}=R_{j k}^{(s)}\left(\theta_{j}\right) W_{i}^{(s)}$. According to Eqs. (4) and (5), the Fresnel reflection coefficient $R_{j k}\left(\theta_{j}\right)$ for incident natural light is

$$
R_{j k}\left(\theta_{j}\right)=\frac{1}{2}\left[R_{j k}^{(p)}\left(\theta_{j}\right)+R_{j k}^{(s)}\left(\theta_{j}\right)\right] .
$$

The reflected light is partially polarized, since the two linearly polarized irradiances $W_{r}^{(p)}$ and $W_{r}^{(s)}$ are different due to the difference between the Fresnel reflection coefficients $R_{j k}^{(p)}\left(\theta_{j}\right)$ and $R_{j k}^{(s)}\left(\theta_{j}\right)$. The reflected light is still incoherent; i.e., the irradiances $W_{r}^{(p)}$ and $W_{r}^{(s)}$ are independent. If the reflected light reaches a new interface, the Fresnel coefficient $R_{j k}\left(\theta_{j}\right)$ expressed in Eq. (6) cannot be applied. However, we may consider separately the reflection of the irradiance $W_{r}^{(p)}$ and the reflection of the irradiance $W_{r}^{(s)}$ and sum the resulting reflected irradiances.

Since the energy is conserved at the interface, the Fresnel transmission coefficients $T_{j k}^{(p)}\left(\theta_{j}\right), T_{j k}^{(s)}\left(\theta_{j}\right)$, and $T_{j k}\left(\theta_{j}\right)$ are related to the Fresnel reflection coefficients $R_{j k}^{(p)}\left(\theta_{j}\right), R_{j k}^{(s)}\left(\theta_{j}\right)$, and $R_{j k}\left(\theta_{j}\right)$, respectively, by

$$
T_{j k}^{*}\left(\theta_{j}\right)=1-R_{j k}^{*}\left(\theta_{j}\right) .
$$

Similarly to the reflected light, the transmitted light is also incoherent and partially polarized; i.e., the two transmitted linearly polarized irradiances are independent but not equal. Therefore, if the incident natural light interacts successively with several interfaces (reflections or transmissions), we have to consider separately the $s$-polarized component and the $p$-polarized component.

For both polarizations, and thereby for natural light, the reflection and transmission coefficients verify the following property,

$$
R_{j k}^{*}\left(\theta_{j}\right)=R_{k j}^{*}\left(\theta_{k}\right),
$$

and consequently 


$$
T_{j k}^{*}\left(\theta_{j}\right)=T_{k j}^{*}\left(\theta_{k}\right) .
$$

A light pencil passing from a medium $m_{j}$ of refractive index $n_{j}$ to a medium $m_{k}$ of refractive index $n_{k}$ is subject to refraction. According to Snell's laws, the refraction modifies the pencil's main direction $\theta_{i}$ into a direction $\theta_{k}$ and its solid angle $\mathrm{d} \Omega_{i}$ into a solid angle $\mathrm{d} \Omega_{k}$, according to the relation ${ }^{11}$

$$
\mathrm{d} \Omega_{k}=\left(\frac{n_{j}}{n_{k}}\right)^{2} \frac{\cos \theta_{j}}{\cos \theta_{k}} \mathrm{~d} \Omega_{j} .
$$

\section{A. Diffuse Reflectance of an Interface}

Let us consider an interface between media of different refractive indices $n_{j}$ and $n_{k}$ illuminated by a Lambertian irradiance $W_{i}$ composed of natural light. The interface receives from all directions $\left(\theta_{j}, \phi_{j}\right)$ of the upper hemisphere a constant radiance $L_{i}=W_{i} / \pi$, composed of a $p$-polarized component $L_{i}^{(p)}$ and an $s$-polarized component $L_{i}^{(s)}$ :

$$
L_{i}^{(p)}=L_{i}^{(s)}=\frac{L_{i}}{2}=\frac{W_{i}}{2 \pi} .
$$

Let us first consider the $p$-polarized component. The element of irradiance $\mathrm{d} W_{i}^{(p)}\left(\theta_{j}, \phi_{j}\right)$ received from a direction $\left(\theta_{j}, \phi_{j}\right)$ is related to the radiance $L_{i}^{(p)}$ by

$$
\mathrm{d} W_{i}^{(p)}\left(\theta_{j}, \phi_{j}\right)=L_{i}^{(p)} \cos \theta_{j} \mathrm{~d} \Omega_{j}=L_{i}^{(p)} \cos \theta_{j} \sin \theta_{j} \mathrm{~d} \theta_{j} \mathrm{~d} \phi_{j} .
$$

This element of irradiance $\mathrm{d} W_{i}^{(p)}\left(\theta_{j}, \phi_{j}\right)$ is reflected by the interface within a proportion $R_{j k}^{(p)}\left(\theta_{j}\right)$ given by the Fresnel formulas. The reflected element of irradiance $\mathrm{d} W_{r}^{(p)}\left(\theta_{j}, \phi_{j}\right)$ is therefore

$$
\mathrm{d} W_{r}^{(p)}\left(\theta_{j}, \phi_{j}\right)=R_{j k}^{(p)}\left(\theta_{j}\right) L_{i}^{(p)} \cos \theta_{j} \sin \theta_{j} \mathrm{~d} \theta_{j} \mathrm{~d} \phi_{j} .
$$

Similarly, the interface receiving the $s$-polarized radiance $L_{i}^{(s)}$ from the same direction from the same direction $\left(\theta_{j}, \phi_{j}\right)$ reflects an element of irradiance $\mathrm{d} W_{r}^{(s)}\left(\theta_{j}, \phi_{j}\right)$ :

$$
\mathrm{d} W_{r}^{(s)}\left(\theta_{j}, \phi_{j}\right)=R_{j k}^{(s)}\left(\theta_{j}\right) L_{i}^{(s)} \cos \theta_{j} \sin \theta_{j} \mathrm{~d} \theta_{j} \mathrm{~d} \phi_{j} .
$$

The total reflected element of irradiance $\mathrm{d} W_{r}\left(\theta_{j}, \phi_{j}\right)$ is the sum of the components $\mathrm{d} W_{r}^{(p)}\left(\theta_{j}, \phi_{j}\right)$ and $\mathrm{d} W_{r}^{(s)}\left(\theta_{j}, \phi_{j}\right)$. Its expression is given by the sum of Eqs. (13) and (14), in which we replace $L_{i}^{(p)}$ and $L_{i}^{(s)}$ with $W_{i} / 2 \pi$ according to Eq. (11), and we replace $R_{j k}^{(p)}\left(\theta_{j}\right)+R_{j k}^{(s)}\left(\theta_{j}\right)$ with $2 R_{j k}\left(\theta_{j}\right)$ according to the definition of the Fresnel reflection factor for natural light, Eq. (6). Therefore,

$$
\mathrm{d} W_{r}\left(\theta_{j}, \phi_{j}\right)=R_{j k}\left(\theta_{j}\right) \frac{W_{i}}{\pi} \cos \theta_{j} \sin \theta_{j} \mathrm{~d} \theta_{j} \mathrm{~d} \phi_{j} .
$$

The total reflected irradiance $W_{r}$ is the sum of the element of irradiance reflected in all directions of the upper hemisphere:

$$
W_{r}=\int_{\phi_{j}=0}^{2 \pi} \int_{\theta_{j}=0}^{\pi / 2} R_{j k}\left(\theta_{j}\right) \frac{W_{i}}{\pi} \cos \theta_{j} \sin \theta_{j} \mathrm{~d} \theta_{j} \mathrm{~d} \phi_{j} .
$$

Since in Eq. (16) the integrated terms do not depend on $\phi_{j}$, the integration according to $\phi_{j}$ yields a factor $2 \pi$. With $2 \cos \theta_{j} \sin \theta_{j}=\sin 2 \theta_{j}$, Eq. (16) becomes

$$
W_{r}=W_{i} \int_{\theta_{j}=0}^{\pi / 2} R_{j k}\left(\theta_{j}\right) \sin 2 \theta_{j} \mathrm{~d} \theta_{j} .
$$

The ratio $W_{r} / W_{i}$ then gives the diffuse reflectance $r_{j k}$ of the interface ${ }^{12}$ :

$$
r_{j k}=\int_{\theta_{j}=0}^{\pi / 2} R_{j k}\left(\theta_{j}\right) \sin 2 \theta_{j} \mathrm{~d} \theta_{j} .
$$

Since the interface does not absorb light and since the energy is conserved, the transmittance $t_{j k}$ of the interface for Lambertian incident light is

$$
t_{j k}=1-r_{j k} \text {. }
$$

\section{B. Attenuation of Light in an Absorbing Medium}

A collimated light flux traversing a path of length $x$ in a nondiffusing absorbing medium, of linear absorption coefficient $\alpha$, is attenuated according to a proportion $t$ given by Beer's law

$$
t=e^{-\alpha x}
$$

\section{REFLECTANCE AND TRANSMITTANCE OF NONSCATTERING SUPERPOSED COLORING LAYERS OF DIFFERENT REFRACTIVE INDICES}

In the present section, we characterize the reflection and transmission properties of a coloring (absorbing) nonscattering layer surrounded by two other media of different refractive indices. The difference of refractive index at the interfaces induces Fresnel reflections. Hence, multiple reflections occur within the considered layer, which increases the light absorption.

The nonscattering layer $m_{1}$ has a refractive index $n_{1}$, a wavelength-dependent absorption coefficient $\alpha_{1}$, and a thickness $h_{1}$. Surrounding media $m_{0}$ and $m_{2}$ have respective refractive indices $n_{0}$ and $n_{2}$. The interface $i_{01}$ between $m_{0}$ and $m_{1}$ and the interface $i_{12}$ between $m_{1}$ and $m_{2}$ are parallel planes. The distance $h_{1}$ between these parallel planes is significantly larger than the wavelengths of light, thereby avoiding interference phenomena (Fig. 2).

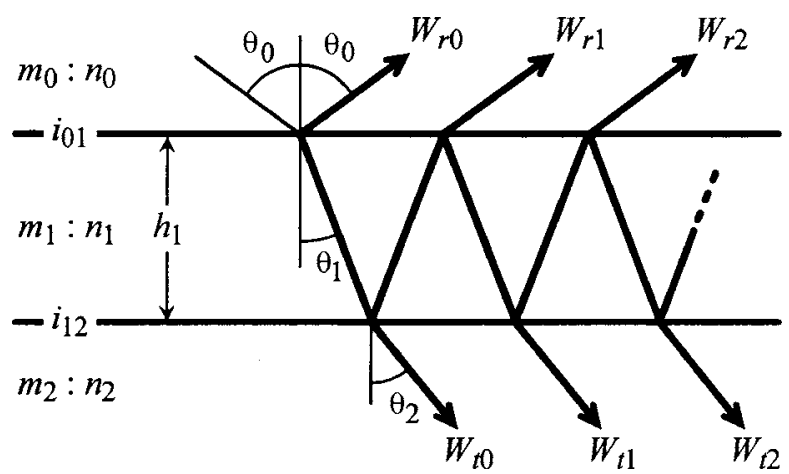

Fig. 2. Reflection and refraction within a nonscattering coloring layer $m_{1}$. 
We distinguish the coloring layer alone, $m_{1}$, considered without its interfaces with the surrounding media, and the coloring layer with its interfaces, $M_{1}$, called the bounded layer. Inside the colored nonscattering layer $m_{1}$, light is either absorbed or transmitted, but not reflected. However, due to multiple reflections at the interfaces with surrounding media of different refractive indices, the bounded layer $M_{1}$ illuminated from medium $m_{0}$ reflects light back to medium $m_{0}$ and transmits light to medium $m_{2}$. The reflectance and the transmittance of the bounded layer $M_{1}$ are expressed according to the polarization of the incident light, according to its geometry (collimated or diffuse), and according to the side from which the bounded layer is illuminated (medium $m_{0}$ or medium $m_{2}$ ).

The incident light is incoherent and unpolarized (natural light), collimated with an incidence angle $\theta_{0}$, and comes from medium $m_{0}$. The incident irradiance is decomposed into a $p$-polarized component and an $s$-polarized component. The $p$ - and $s$-polarized components are reflected by the bounded layer, with proportions $R_{012}^{(p)}\left(\theta_{0}\right)$ and $R_{012}^{(s)}\left(\theta_{0}\right)$, respectively, and transmitted with proportions $T_{012}^{(p)}\left(\theta_{0}\right)$ and $T_{012}^{(s)}\left(\theta_{0}\right)$, respectively. The global reflectance and transmittance of the bounded layer illuminated by natural light is called $R_{012}\left(\theta_{0}\right)$, and its transmittance is called $T_{012}\left(\theta_{0}\right)$. For a Lambertian illumination from medium $m_{0}$, the bounded layer's reflectance and transmittance are called, respectively, $r_{012}$ and $t_{012}$.

Let us express each of these reflectances and transmittances. They depend on the refractive indices $n_{0}, n_{1}$, and $n_{2}$, the coloring layer's thickness $h_{1}$ and its wavelengthdependent absorption coefficient $\alpha_{1}$.

\section{A. Reflectance and Transmittance of the Bounded Coloring Layer for Collimated Incident Light}

A collimated incoherent and unpolarized light (natural light) illuminates the bounded layer $M_{1}$ from medium $m_{0}$ with an angle $\theta_{0}$ (see Fig. 2). The incident irradiance $W_{i}$ is decomposed into a $p$-polarized irradiance $W_{i}^{(p)}$ and an $s$-polarized irradiance $W_{i}^{(s)}$, with

$$
W_{i}^{(p)}=W_{i}^{(s)}=W_{i} / 2 .
$$

The components $W_{i}^{(p)}$ and $W_{i}^{(s)}$ are subjected to multiple reflections within the bounded layer $M_{1}$. To describe these multiple reflections, we calculate the reflected irradiances $W_{r}^{(p)}$ and $W_{r}^{(s)}$ and the transmitted irradiances $W_{t}^{(p)}$ and $W_{t}^{(s)}$. We then derive the reflectance and the transmittance of the bounded layer for the $p$-polarized component, the $s$-polarized component, and finally for natural incident light.

The phenomenon of multiple reflections is identical for the two components $W_{i}^{(p)}$ and $W_{i}^{(s)}$, with different Fresnel coefficients with respect to their respective polarizations. Hence, the interaction of polarized irradiances $W_{i}^{(p)}$ and $W_{i}^{(s)}$ with the bounded layer is presented only once, with a superscript ${ }^{*}$ representing either superscript $(p)$ or superscript $(s)$.

A portion $R_{01}^{*}\left(\theta_{0}\right)$ of the polarized incident irradiance $W_{i}^{*}$ is reflected by the interface $i_{01}$. It propagates along the specular direction, i.e., at an angle $\theta_{0}$. The reflected ir- radance $W_{r 0}^{*}$ is the first contribution to the total polarizaed reflected irradiance $W_{r}^{*}$ :

$$
W_{r 0}^{*}=R_{01}^{*}\left(\theta_{0}\right) W_{i}^{*}
$$

A portion $T_{01}^{*}\left(\theta_{0}\right)$ of $W_{i}^{*}$ is transmitted into layer $m_{1}$ at an angle $\theta_{1}$, crosses the layer along a path of length $h_{1} / \cos \theta_{1}$, and is attenuated by a factor $t_{1}\left(\theta_{1}\right)$ due to absorption (Beer's law):

$$
t_{1}\left(\theta_{1}\right)=e^{-\alpha_{1} h_{1} / \cos \theta_{1}} .
$$

The irradiance reaching the interface $i_{12}$ is therefore $T_{01}^{*}\left(\theta_{0}\right) t_{1}\left(\theta_{1}\right) W_{i}^{*}$. A portion $R_{12}^{*}\left(\theta_{1}\right)$ of this irradiance is internally reflected by the interface $i_{12}$, and a portion $T_{12}^{*}\left(\theta_{1}\right)$ is transmitted into medium 2 at an angle $\theta_{2}$. According to Snell's law,

$$
n_{0} \sin \theta_{0}=n_{1} \sin \theta_{1}=n_{2} \sin \theta_{2} .
$$

The irradiance $W_{r 0}^{*}$ is the first contribution to the total transmitted irradiance $W_{t}^{*}$ :

$$
W_{t 0}^{*}=T_{01}^{*}\left(\theta_{0}\right) T_{12}^{*}\left(\theta_{1}\right) t_{1}\left(\theta_{1}\right) W_{i}^{*} .
$$

The irradiance $T_{01}^{*}\left(\theta_{0}\right) R_{12}^{*}\left(\theta_{1}\right) t_{1}\left(\theta_{1}\right) W_{i}^{*}$ that is internally reflected by the interface $i_{12}$ again crosses layer $m_{1}$ [attenuation factor $t_{1}\left(\theta_{1}\right)$ due to absorption] and is either internally reflected by the interface $i_{01}$ [Fresnel reflection factor $R_{10}^{*}\left(\theta_{1}\right)$ ] or transmitted [Fresnel transmission factor $T_{10}^{*}\left(\theta_{1}\right)$ ] across that interface $i_{01}$. The irradiance $W_{r 1}^{*}$, emerging from the interface $i_{01}$, is the second contribution to the total reflected irradiance $W_{r}^{*}$ :

$$
W_{r 1}^{*}=T_{01}^{*}\left(\theta_{0}\right) R_{12}^{*}\left(\theta_{1}\right) T_{10}^{*}\left(\theta_{1}\right) t_{1}^{2}\left(\theta_{1}\right) W_{i}^{*} .
$$

Then, owing to the multiple internal reflections, the light alternately crosses layer $m_{1}$ toward interfaces $i_{01}$ and $i_{12}$. Along the same line of reasoning, we obtain obtained successive expressions of reflected in irradiances $W_{r k}^{*}$ and transmitted irradiances $W_{t k}^{*}$. The sum of these irradiances forms respectively the total reflected irradiance $W_{r}^{*}$ and the total transmitted iradiance irradiance $W_{t}^{*}$.

Let us first calculate the total reflected irradiance irradinace $W_{r}^{*}$. The irradiances $W_{r k}^{*}, k=1,2 \ldots$, are internally reflected $k$ times by the interface $i_{12}$ and $k-1$ times by the interface $i_{10}$. They cross they the layer $m_{1} 2 k$ times. Therefore, the generic expression of $W_{r k}^{*}$, for $k \geqslant 1$ is

$$
W_{r k}^{*}=T_{01}^{*}\left(\theta_{0}\right)\left[R_{10}^{*}\left(\theta_{1}\right)\right]^{k-1}\left[R_{12}^{*}\left(\theta_{1}\right)\right]^{k} t_{1}^{2 k}\left(\theta_{1}\right) T_{10}^{*}\left(\theta_{1}\right) W_{i}^{*} .
$$

The total polarized reflected irradiance $W_{r}^{*}$, which emerges into medium $m_{0}$ with an angle $\theta_{0}$, results from the sum of all the reflected irradiances $W_{r k}^{*}$ :

$$
\begin{aligned}
W_{r}^{*}= & R_{01}^{*}\left(\theta_{0}\right) W_{i}^{*} \\
& +\frac{T_{01}^{*}\left(\theta_{0}\right) T_{10}^{*}\left(\theta_{1}\right)}{R_{10}^{*}\left(\theta_{1}\right)} W_{i}^{*} \sum_{k=1}^{\infty}\left[R_{10}^{*}\left(\theta_{1}\right) R_{12}^{*}\left(\theta_{1}\right) t_{1}^{2}\left(\theta_{1}\right)\right]^{k} .
\end{aligned}
$$

The infinite sum is a geometrical series. Since $T_{10}^{*}\left(\theta_{1}\right)$ $=T_{01}^{*}\left(\theta_{0}\right)$ according to relation (9), Eq. (28) becomes 


$$
W_{r}^{*}=R_{01}^{*}\left(\theta_{0}\right) W_{i}^{*}+\frac{\left[T_{10}^{*}\left(\theta_{1}\right)\right]^{2} R_{12}^{*}\left(\theta_{1}\right) t_{1}^{2}\left(\theta_{1}\right)}{1-R_{10}^{*}\left(\theta_{1}\right) R_{12}^{*}\left(\theta_{1}\right) t_{1}^{2}\left(\theta_{1}\right)} W_{i}^{*} .
$$

The ratio $W_{r}^{*} / W_{i}^{*}$ gives the reflectance $R_{012}^{*}\left(\theta_{0}\right)$ of the bounded layer $M_{1}$ illuminated at an incidence $\theta_{0}$ by a $p$-polarized or an $s$-polarized collimated incident light:

$$
R_{012}^{*}\left(\theta_{0}\right)=R_{01}^{*}\left(\theta_{0}\right)+\frac{\left[T_{10}^{*}\left(\theta_{1}\right)\right]^{2} R_{12}^{*}\left(\theta_{1}\right) t_{1}^{2}\left(\theta_{1}\right)}{1-R_{10}^{*}\left(\theta_{1}\right) R_{12}^{*}\left(\theta_{1}\right) t_{1}^{2}\left(\theta_{1}\right)} .
$$

For natural incident light, the total reflected irradiance $W_{r}$ is given by the sum of the resulting $p$-polarized and $s$-polarized reflected irradiances given by Eq. (29):

$$
W_{r}=W_{r}^{(p)}+W_{r}^{(s)}=R_{012}^{(p)}\left(\theta_{0}\right) W_{i}^{(p)}+R_{012}^{(s)}\left(\theta_{0}\right) W_{i}^{(s)} .
$$

By replacing $W_{i}^{(p)}$ and $W_{i}^{(s)}$ with $W_{i} / 2$ according to Eq. (21), we obtain

$$
W_{r}=\frac{1}{2}\left[R_{012}^{(p)}\left(\theta_{0}\right)+R_{012}^{(s)}\left(\theta_{0}\right)\right] W_{i} .
$$

The ratio $W_{r} / W_{i}$ gives the reflectance $R_{012}\left(\theta_{0}\right)$ of the bounded layer $M_{1}$ surrounded by media $m_{0}$ and $m_{2}$, illuminated from $m_{0}$ by collimated natural light at an incidence $\theta_{0}$ :

$$
R_{012}\left(\theta_{0}\right)=\frac{1}{2}\left[R_{012}^{(p)}\left(\theta_{0}\right)+R_{012}^{(s)}\left(\theta_{0}\right)\right] .
$$

Let us now calculate the total transmitted irradiance $W_{t}^{*}$ and derive the expression of the transmittance of the bounded layer for $p$-polarized, $s$-polarized, and natural incident light. The irradiances $W_{t k}^{*}$ are internally reflected $k$ times by the interface $i_{12}$ and $k$ times by the interface $i_{01}$. They cross the layer $m_{1} 2 k+1$ times. The generic expression of $W_{t k}^{*}$ for $k \geqslant 0$ is

$$
W_{t k}^{*}=T_{01}^{*}\left(\theta_{0}\right)\left[R_{10}^{*}\left(\theta_{1}\right)\right]^{k}\left[R_{12}^{*}\left(\theta_{1}\right)\right]^{k}\left[t_{1}\left(\theta_{1}\right)\right]^{2 k+1} T_{12}^{*}\left(\theta_{1}\right) W_{i}^{*} .
$$

The total transmitted irradiance $W_{t}^{*}$ that emerges into medium $m_{2}$ at an angle $\theta_{2}$ results from the sum of all the transmitted irradiances $W_{t k}^{*}$,

$$
W_{t}^{*}=T_{01}^{*}\left(\theta_{0}\right) T_{12}^{*}\left(\theta_{1}\right) t_{1}\left(\theta_{1}\right) W_{i}^{*} \sum_{k=0}^{\infty}\left[R_{10}^{*}\left(\theta_{1}\right) R_{12}^{*}\left(\theta_{1}\right) t_{1}^{2}\left(\theta_{1}\right)\right]^{k},
$$

where the infinite sum is a converging geometric series. Relation (35) becomes

$$
W_{t}^{*}=\frac{T_{01}^{*}\left(\theta_{0}\right) T_{12}^{*}\left(\theta_{1}\right) t_{1}\left(\theta_{1}\right)}{1-R_{10}^{*}\left(\theta_{1}\right) R_{12}^{*}\left(\theta_{1}\right) t_{1}^{2}\left(\theta_{1}\right)} W_{i}^{*} .
$$

The ratio $W_{t}^{*} / W_{i}^{*}$ gives the reflectance $T_{012}^{*}\left(\theta_{0}\right)$ of the bounded layer $M_{1}$ illuminated under an incidence $\theta_{0}$ by a $p$-polarized or an $s$-polarized collimated incident light:

$$
T_{012}^{*}\left(\theta_{0}\right)=\frac{T_{01}^{*}\left(\theta_{0}\right) T_{12}^{*}\left(\theta_{1}\right) t_{1}\left(\theta_{1}\right)}{1-R_{10}^{*}\left(\theta_{1}\right) R_{12}^{*}\left(\theta_{1}\right) t_{1}^{2}\left(\theta_{1}\right)} .
$$

For natural incident light, the total transmitted irradiance $W_{t}$ is given by the sum of the $p$-polarized and the $s$-polarized transmitted irradiances $W_{t}^{(p)}$ and $W_{t}^{(s)}$, given by Eq. (36). We replace $W_{i}^{(p)}$ and $W_{i}^{(s)}$ by $W_{i} / 2$ according to Eq. (21) and obtain

$$
W_{t}=\frac{1}{2}\left[T_{012}^{(p)}\left(\theta_{0}\right)+T_{012}^{(s)}\left(\theta_{0}\right)\right] W_{i} .
$$

The ratio $W_{t} / W_{i}$ gives the transmittance $T_{012}\left(\theta_{0}\right)$ of the bounded layer $M_{1}$ surrounded with media $m_{0}$ and $m_{2}$, illuminated from $m_{0}$ by a collimated natural light with an incidence angle $\theta_{0}$ :

$$
T_{012}\left(\theta_{0}\right)=\frac{1}{2}\left[T_{012}^{(p)}\left(\theta_{0}\right)+T_{012}^{(s)}\left(\theta_{0}\right)\right] .
$$

Let us now assume that the collimated light comes from $m_{2}$ at an incidence angle $\theta_{2}$. The description of the multiple reflections occurring within the bounded layer is the same as when the light comes from medium $m_{0}$. We obtain the same expressions of reflectance and transmittance as above with exchanged subscripts 0 and 2. For the $p$-polarized or the $s$-polarized incident irradiance components, the bounded layer's reflectance $R_{210}^{*}\left(\theta_{2}\right)$ is

$$
R_{210}^{*}\left(\theta_{2}\right)=R_{21}^{*}\left(\theta_{2}\right)+\frac{\left[T_{12}^{*}\left(\theta_{1}\right)\right]^{2} R_{10}^{*}\left(\theta_{1}\right) t_{1}^{2}\left(\theta_{1}\right)}{1-R_{12}^{*}\left(\theta_{1}\right) R_{10}^{*}\left(\theta_{1}\right) t_{1}^{2}\left(\theta_{1}\right)} .
$$

For natural incident light, the bounded layer's reflectance $R_{210}\left(\theta_{2}\right)$ is

$$
R_{210}\left(\theta_{2}\right)=\frac{1}{2}\left[R_{210}^{(p)}\left(\theta_{2}\right)+R_{210}^{(s)}\left(\theta_{2}\right)\right],
$$

where $R_{210}^{(p)}\left(\theta_{2}\right)$ and $R_{210}^{(s)}\left(\theta_{2}\right)$ are given by Eq. (40). Similarly, for the $p$-polarized or the $s$-polarized incident irradiance components, the bounded layer's transmittance $T_{210}^{*}\left(\theta_{2}\right)$ is

$$
T_{210}^{*}\left(\theta_{2}\right)=\frac{T_{21}^{*}\left(\theta_{2}\right) T_{10}^{*}\left(\theta_{1}\right) t_{1}\left(\theta_{1}\right)}{1-R_{12}^{*}\left(\theta_{1}\right) R_{10}^{*}\left(\theta_{1}\right) t_{1}^{2}\left(\theta_{1}\right)},
$$

and for natural incident light, its transmittance $T_{210}\left(\theta_{2}\right)$ is

$$
T_{210}\left(\theta_{2}\right)=\frac{1}{2}\left[T_{210}^{(p)}\left(\theta_{2}\right)+T_{210}^{(s)}\left(\theta_{2}\right)\right] .
$$

Since $T_{10}^{*}\left(\theta_{1}\right)=T_{01}^{*}\left(\theta_{0}\right)$ and $T_{12}^{*}\left(\theta_{1}\right)=T_{21}^{*}\left(\theta_{2}\right)$ according to relation (9), we observe that expressions (37) and (42) are identical. Thus, for the linearly polarized components, and thereby for natural light, the transmittance of the bounded layer $M_{1}$ does not depend on the orientation of light propagation:

$$
T_{210}\left(\theta_{2}\right)=T_{012}\left(\theta_{0}\right) .
$$

The bounded layer's reflectance depends on the orientation of light propagation only in respect to the Fresnel reflection on the first interface encountered by the incident light, i.e., $R_{01}^{*}\left(\theta_{0}\right)$ in Eq. (30) and $R_{21}^{*}\left(\theta_{2}\right)$ in Eq. (40).

\section{B. Diffuse Reflectance of the Bounded Coloring Layer}

The diffuse reflectance $r_{012}$ of the bounded coloring layer $M_{1}$ surrounded by nonscattering media $m_{0}$ and $m_{2}$ gives the fraction of incident Lambertian irradiance coming from medium $m_{0}$ that is reflected by the layer back to medium $m_{0}$.

The incident light is diffuse, incoherent, and unpolarized. It constitutes a Lambertian irradiance $W_{i}$, composed 
of elements of irradiance $\mathrm{d} W_{i}\left(\theta_{0}, \phi_{0}\right)$ that can be expressed as a function of the direction-independent radiance $W_{i} / \pi$ (see Section 2):

$$
\mathrm{d} W_{i}\left(\theta_{0}, \phi_{0}\right)=\frac{W_{i}}{\pi} \cos \theta_{0} \sin \theta_{0} \mathrm{~d} \theta_{0} \mathrm{~d} \phi_{0} .
$$

Each element of irradiance $\mathrm{d} W_{i}\left(\theta_{0}, \phi_{0}\right)$ is reflected by the bounded layer $M_{1}$ with a proportion $R_{012}\left(\theta_{0}\right)$ given by Eq. (33). The term $R_{012}\left(\theta_{0}\right)$ accounts for the multiple reflections undergone by both linearly polarized components within the bounded layer $M_{1}$. The sum of all the reflected elements of irradiance $R_{012}\left(\theta_{0}\right) d W_{i}\left(\theta_{0}, \phi_{0}\right)$, performed over the all incidence angles $\left(\theta_{0}, \phi_{0}\right)$ of the hemisphere, yields the total reflected irradiance $W_{r}$ :

$$
W_{r}=\int_{\phi_{0}=0}^{2 \pi} \int_{\theta_{0}=0}^{\pi / 2} R_{012}\left(\theta_{0}\right) \frac{W_{i}}{\pi} \cos \theta_{0} \sin \theta_{0} \mathrm{~d} \theta_{0} \mathrm{~d} \phi_{0} .
$$

Since the integrated terms do not depend on the azimuth angle $\phi_{0}$, the integration according to $\phi_{0}$ yields a factor $2 \pi$. After rearranging the expression of $W_{r}$ in the same manner as Eq. (17), the ratio $W_{r} / W_{i}$ yields the diffuse reflectance $r_{012}$ of the bounded layer $M_{1}$, surrounded by media $m_{0}$ and $m_{2}$ and illuminated from $m_{0}$ by diffuse light:

$$
r_{012}=\int_{\theta_{0}=0}^{\pi / 2} R_{012}\left(\theta_{0}\right) \sin 2 \theta_{0} \mathrm{~d} \theta_{0} .
$$

This expression generalizes the diffuse reflectance $r_{01}$ of an interface, Eq. (18), to a coloring nonscattering layer that has a refractive index different from its surrounding media.

C. Diffuse Transmittance of the Bounded Coloring Layer The diffuse transmittance $t_{012}$ of the bounded coloring layer $M_{1}$ surrounded by nonscattering media $m_{0}$ and $m_{2}$ gives the fraction of incident Lambertian irradiance coming from medium $m_{0}$ that is transmitted across the layer and therefore emerges into medium $m_{2}$.

Each incident element of irradiance $\mathrm{d} W_{i}\left(\theta_{0}, \phi_{0}\right)$ expressed in relation (45) is transmitted across the bounded layer $M_{1}$ with a proportion $T_{012}\left(\theta_{0}\right)$ given by Eq. (39). The sum of all transmitted elements of irradiance $T_{012}\left(\theta_{0}\right) \mathrm{d} E_{i}\left(\theta_{0}, \phi_{0}\right)$, performed over all the incidence angles $\left(\theta_{0}, \phi_{0}\right)$ of the hemisphere, yields the transmitted irradiance $W_{t}$ :

$$
W_{t}=\int_{\phi_{0}=0}^{2 \pi} \int_{\theta_{0}=0}^{\pi / 2} T_{012}\left(\theta_{0}\right) \frac{W_{i}}{\pi} \cos \theta_{0} \sin \theta_{0} \mathrm{~d} \theta_{0} \mathrm{~d} \phi_{0} .
$$

After applying the same simplifications as previously, we obtain the diffuse transmittance of the bounded coloring layer $M_{1}$ :

$$
t_{012}=\frac{W_{t}}{W_{i}}=\int_{\theta_{0}=0}^{\pi / 2} T_{012}\left(\theta_{0}\right) \sin 2 \theta_{0} \mathrm{~d} \theta_{0} .
$$

This expression generalizes the diffuse transmittance of $t_{01}$ of an interface to an absorbing layer that has an index of refraction different from air. However, since the bounded coloring layer $M_{1}$ absorbs light, the energy is not conserved and $t_{012}$ is different from $1-r_{012}$.

\section{Reflectance and Transmittance of Two or More Superposed Nonscattering Bounded Coloring Layers} Two nonscattering coloring layers $m_{1}$ and $m_{2}$, of respective refractive indices $n_{1}$ and $n_{2}$, thicknesses $h_{1}$ and $h_{2}$, and absorption coefficients $\alpha_{1}$ and $\alpha_{2}$, are superposed and surrounded by nonscattering media $m_{0}$ and $m_{3}$. The three interfaces $i_{01}, i_{12}$, and $i_{23}$ are parallel planes.

The reflectance and transmittance of the two superposed bounded layers, for collimated illumination from $m_{0}$ with an incidence angle $\theta_{0}$, are called, respectively, $R_{0123}\left(\theta_{0}\right)$ and $T_{0123}\left(\theta_{0}\right)$. As previously, the incident light is incoherent and unpolarized.

The reflectance $R_{0123}\left(\theta_{0}\right)$ of two superposed bounded layers is the average of the two reflectances obtained for the $p$-polarized and the $s$-polarized components:

$$
R_{0123}\left(\theta_{0}\right)=\frac{1}{2}\left[R_{0123}^{(p)}\left(\theta_{0}\right)+R_{0123}^{(s)}\left(\theta_{0}\right)\right] .
$$

$R_{0123}^{(p)}\left(\theta_{0}\right)$ and $R_{0123}^{(s)}\left(\theta_{0}\right)$ are extensions of the reflectances $R_{012}^{(p)}\left(\theta_{0}\right)$ and $R_{012}^{(s)}\left(\theta_{0}\right)$ of a single coloring layer $M_{1}$ surrounded by media $m_{0}$ and $m_{2}$ [Eq. (30)]. When deriving the expression of $R_{012}^{*}\left(\theta_{0}\right)$, we considered the multiple reflections within the layer $m_{1}$ between the interfaces $i_{01}$ [Fresnel reflection coefficient $R_{10}^{*}\left(\theta_{1}\right)$ ] and $i_{12}$ [Fresnel reflection coefficient $R_{12}^{*}\left(\theta_{1}\right)$ ]. In the present case, we consider the multiple reflections within layer $m_{1}$ between the interfaces $i_{01}$ [Fresnel reflection coefficient $R_{10}^{*}\left(\theta_{1}\right)$ ] and the bounded layer $M_{2}$ surrounded by media $m_{1}$ and $m_{3}$, of reflectance $R_{123}^{*}\left(\theta_{1}\right)$. The expression of $R_{0123}^{*}\left(\theta_{0}\right)$ therefore derives from the expression of $R_{012}^{*}\left(\theta_{0}\right)$, in which $R_{12}^{*}\left(\theta_{1}\right)$ is replaced by $R_{123}^{*}\left(\theta_{1}\right)$ :

$$
R_{0123}^{*}\left(\theta_{0}\right)=R_{01}^{*}\left(\theta_{0}\right)+\frac{\left[T_{01}^{*}\left(\theta_{0}\right)\right]^{2} R_{123}^{*}\left(\theta_{1}\right) t_{1}^{2}(\theta)}{1-R_{10}^{*}\left(\theta_{1}\right) R_{123}^{*}\left(\theta_{1}\right) t_{1}^{2}(\theta)} .
$$

The thickness $h_{2}$ and the absorption coefficient $\alpha_{2}$ of the layer $m_{2}$ are implicit within the term $R_{123}\left(\theta_{1}\right)$.

Similar considerations apply for the transmittance $T_{0123}\left(\theta_{0}\right)$ :

$$
T_{0123}\left(\theta_{0}\right)=\frac{1}{2}\left[T_{0123}^{(p)}\left(\theta_{0}\right)+T_{0123}^{(s)}\left(\theta_{0}\right)\right],
$$

where the expressions $T_{0123}^{(p)}\left(\theta_{0}\right)$ and $T_{0123}^{(s)}\left(\theta_{0}\right)$ derive from the expressions $T_{012}^{(p)}\left(\theta_{0}\right)$ and $T_{012}^{(s)}\left(\theta_{0}\right)$ by replacing $T_{12}^{*}\left(\theta_{1}\right)$ with $T_{123}^{*}\left(\theta_{1}\right)$ in Eq. (37):

$$
T_{0123}^{*}\left(\theta_{0}\right)=\frac{T_{01}^{*}\left(\theta_{0}\right) T_{123}^{*}\left(\theta_{1}\right) t_{1}\left(\theta_{1}\right)}{1-R_{10}^{*}\left(\theta_{1}\right) R_{123}^{*}\left(\theta_{1}\right) t_{1}^{2}\left(\theta_{1}\right)} .
$$

By following the same line of reasoning but starting with the reflectance and transmittance of the bounded layer $M_{2}$ instead of the reflectance and transmittance of the bounded layer $M_{1}$, we obtain expressions that are exactly equivalent to expressions (51) and (53):

$$
R_{0123}^{*}\left(\theta_{0}\right)=R_{012}^{*}\left(\theta_{0}\right)+\frac{\left[T_{012}^{*}\left(\theta_{0}\right)\right]^{2} R_{23}^{*}\left(\theta_{2}\right) t_{2}^{2}\left(\theta_{2}\right)}{1-R_{210}^{*}\left(\theta_{1}\right) R_{23}^{*}\left(\theta_{2}\right) t_{2}^{2}\left(\theta_{2}\right)}
$$




$$
T_{0123}^{*}\left(\theta_{0}\right)=\frac{T_{012}^{*}\left(\theta_{0}\right) T_{23}^{*}\left(\theta_{2}\right) t_{2}\left(\theta_{2}\right)}{1-R_{210}^{*}\left(\theta_{1}\right) R_{23}^{*}\left(\theta_{2}\right) t_{2}^{2}\left(\theta_{2}\right)}
$$

with

$$
t_{2}\left(\theta_{2}\right)=e^{-\alpha_{2} h_{2} / \cos \theta_{2}} .
$$

Along this line of reasoning, we express the reflectance and transmittance of $k$ superposed bounded coloring layers $M_{1}, \ldots, M_{k}$ surrounded by media $m_{0}$ and $m_{k+1}$ :

$$
\begin{aligned}
& R_{0 \ldots k+1}\left(\theta_{0}\right)=\frac{1}{2}\left[R_{0 \ldots k+1}^{(p)}\left(\theta_{0}\right)+R_{0 \ldots k+1}^{(s)}\left(\theta_{0}\right)\right], \\
& T_{0 \ldots k+1}\left(\theta_{0}\right)=\frac{1}{2}\left[T_{0 \ldots k+1}^{(p)}\left(\theta_{0}\right)+T_{0 \ldots k+1}^{(s)}\left(\theta_{0}\right)\right] .
\end{aligned}
$$

The resulting expressions of $R_{0 \ldots k+1}^{*}\left(\theta_{0}\right)$ and $T_{0 \ldots k+1}^{*}\left(\theta_{0}\right)$ generalize Eqs. (54) and (55) to $k$ stacked nonscattering coloring layers with distinct refractive indices:

$$
R_{0 \ldots k+1}^{*}\left(\theta_{0}\right)=R_{0 \ldots k}^{*}\left(\theta_{0}\right)+\frac{\left[T_{0 \ldots k}^{*}\left(\theta_{0}\right)\right]^{2} R_{k, k+1}^{*}\left(\theta_{k}\right) t_{k}^{2}\left(\theta_{k}\right)}{1-R_{k \ldots 0}^{*}\left(\theta_{k}\right) R_{k, k+1}^{*}\left(\theta_{k}\right) t_{k}^{2}\left(\theta_{k}\right)}
$$

and

$$
T_{0 \ldots k+1}^{*}\left(\theta_{0}\right)=\frac{T_{0 \ldots k}^{*}\left(\theta_{0}\right) T_{k, k+1}^{*}\left(\theta_{k}\right) t_{k}\left(\theta_{k}\right)}{1-R_{k \ldots 0}^{*}\left(\theta_{k}\right) R_{k, k+1}^{*}\left(\theta_{k}\right) t_{k}^{2}\left(\theta_{k}\right)},
$$

with

$$
t_{k}\left(\theta_{k}\right)=e^{-\alpha_{k} h_{k} / \cos \theta_{k}} .
$$

If the incident light is Lambertian, we integrate expressions (57) and (58) over the hemisphere, as in the case of Eqs. (47) and (49), and obtain the diffuse reflectance $r_{0 \ldots k+1}$ and transmittance $t_{0 \ldots k+1}$ of the $k$ superposed bounded coloring layers $M_{1}, \ldots, M_{k}$ surrounded by media $m_{0}$ and $m_{k+1}$ :

$$
r_{0 \ldots k+1}=\int_{\theta_{0}=0}^{\pi / 2} R_{0 \ldots k+1}\left(\theta_{0}\right) \sin 2 \theta_{0} \mathrm{~d} \theta_{0}
$$

and

$$
t_{0 \ldots k+1}=\int_{\theta_{0}=0}^{\pi / 2} T_{0 \ldots k+1}\left(\theta_{0}\right) \sin 2 \theta_{0} \mathrm{~d} \theta_{0} .
$$

\section{REFLECTANCE OF NONSCATTERING COLORING LAYERS SUPERPOSED ON TOP OF A DIFFUSING MEDIUM}

In the previous section, the reflectance and transmittance of stacked nonscattering layers have been expressed, both for collimated and for diffuse incident light, and for natural light or linearly polarized light. We now consider that the stacked layers are surrounded on one side by a transparent nonscattering medium and on the other side by a diffusing background. Each medium may have a distinct refractive index.

We express the reflectance of the background coated with colored nonscattering layers, i.e., the fraction of the incident irradiance that emerges from the interface $i_{01}$. The incident light is assumed to be collimated. The de- rived reflectance expressions are compatible with reflectance measurements performed with an integrating sphere by reference to the reflectance of a perfectly white diffuse reflector. For measurements performed with a radiance detector, we develop the expression of a reflectance factor, which takes into account the geometry of the capturing device.

First, we consider a single colored nonscattering layer $m_{1}$ of refractive index $n_{1}$, thickness $h_{1}$ and absorption coefficient $\alpha_{1}$, surrounded on one side by a nonscattering medium $m_{0}$ of refractive index $n_{0}$, and on the other side by a diffusing background $m_{2}$ of refractive index $n_{2}$. The background is assumed to be a Lambertian reflector; i.e., it reflects a perfectly diffuse and unpolarized light. It is characterized by its wavelength-dependent intrinsic reflectance $\rho_{g}$.

After having established the reflectance of a single bounded nonscattering coloring layer $M_{1}$, we can replace it by any superposition of $k$ bounded nonscattering coloring layers $M_{1}, \ldots, M_{k}$. In the special case where layer $m_{1}$ is surrounded on one side by a reflecting background of identical refractive index and on the other side by air, we obtain the classical Williams-Clapper model. ${ }^{2}$

A. Background Coated with One Nonscattering Layer A collimated irradiance $W_{i}$ (natural light) illuminates the interface $i_{01}$ at an angle $\theta_{0}$. It crosses the bounded coloring layer $M_{1}$ with an attenuation factor $T_{012}\left(\theta_{0}\right)$ [Eq. (39)] and penetrates into the diffusing background, where it is diffused, with a portion $\rho_{g}$ being reflected. The reflected Lambertian irradiance $w_{1}$ is

$$
w_{1}=\rho_{g} T_{012}\left(\theta_{0}\right) W_{i} .
$$

The reflected irradiance $w_{1}$ is unpolarized, owing to multiple scattering within the diffusing background, and can be assimilated to natural light. The bounded layer $M_{1}$ transmits a portion of $w_{1}$ into medium $m_{0}$ and reflects a portion $r_{210}$ [Eq. (47)] toward the background $m_{2}$. The background reflects back toward the bounded layer $m_{1}$ a Lambertian irradiance $w_{2}$ :

$$
w_{2}=\rho_{g} r_{210} w_{1}=\left(\rho_{g} r_{210}\right) \rho_{g} T_{012}\left(\theta_{0}\right) W_{i}
$$

The diffuse light is alternately reflected by the bounded layer $M_{1}$ and the background $m_{2}$. Since the background emits an irradiance $\left(\rho_{g} r_{210}\right)^{k} \rho_{g} T_{012}\left(\theta_{0}\right) W_{i}$ at each internal reflection $k=0,1,2 \ldots$, the total irradiance $W_{g}$ emitted by the background toward the bounded layer $M_{1}$ is

$$
W_{g}=\sum_{k=0}^{\infty}\left(\rho_{g} r_{210}\right)^{k} \rho_{g} T_{012}\left(\theta_{0}\right) W_{i} .
$$

The infinite sum yields a geometrical series that converges toward

$$
W_{g}=\frac{\rho_{g} T_{012}\left(\theta_{0}\right)}{1-\rho_{g} r_{210}} W_{i} .
$$

The irradiance $W_{r}$ that emerges into medium $m_{0}$ results from the transmission of the irradiance $W_{g}$ across the bounded layer $M_{1}$. Since the bounded layer $M_{1}$ has a diffuse transmittance $t_{210}$, similar to that defined in Eq. (49), the emerging irradiance is 


$$
W_{r}=t_{210} \frac{\rho_{g} T_{012}\left(\theta_{0}\right)}{1-\rho_{g} r_{210}} W_{i} .
$$

The reflectance $R_{m 1}$ of the background coated with the bounded layer $m_{1}$ is given by the ratio $W_{r} / W_{i}$ :

$$
R_{m 1}=t_{210} \frac{\rho_{g} T_{012}\left(\theta_{0}\right)}{1-\rho_{g} r_{210}} .
$$

\section{B. Reflectance Factors}

Often, the incident irradiance is not directly accessible. However, it is possible to measure it indirectly with a white reference support, whose reflectance spectrum is known and is generally close to 1 for all the wavelengths of the visible range. The irradiance $W_{r}$ reflected by the sample and the irradiance $W_{\text {ref }}$ reflected by the white reference support are captured by the same measuring device under the same illumination conditions. The ratio $W_{r} / W_{\text {ref }}$ is called the reflectance factor.

The reflectance factor also depends on the geometry of the measuring device. An integrating sphere captures the reflected irradiance $W_{r}$ completely. If the white reference support has a reflectance equal to 1 , we have $W_{\text {ref }}=W_{i}$, and the expressions for the reflectance factor $W_{r} / W_{\text {ref }}$ and the reflectance are identical.

The reflectance factor and the reflectance have different expressions if the capturing device is a radiance detector. The radiance detector does not capture the total reflected irradiance $W_{r}$ but only the radiance $L_{r}\left(\theta_{0}^{\prime}\right)$ reflected in the direction $\theta_{0}^{\prime}$ of the detector. This radiance $L_{r}\left(\theta_{0}^{\prime}\right)$ is defined by the flux emerging from a surface element $\mathrm{d} s$ of the sample within a solid angle $\mathrm{d} \Omega_{0}$ :

$$
L_{r}\left(\theta_{0}^{\prime}\right)=\frac{\mathrm{d}^{2} \Phi}{\mathrm{d} s \cos \theta_{0} \mathrm{~d} \Omega_{0}} .
$$

Let us consider the case of a diffusing background coated with a single nonscattering coloring layer $m_{1}$. We have shown that, owing to the multiple internal reflections, the background emits a total irradiance $W_{g}$, expressed in Eq. (67). We now derive, using the rules of radiometry, ${ }^{11}$ the relation between the irradiance $W_{g}$ and the radiance $L_{r}\left(\theta_{0}^{\prime}\right)$ captured by the radiance detector.

The flux $\mathrm{d}^{2} \Phi$ captured in the direction $\theta_{0}^{\prime}$ corresponds to a collimated flux $\mathrm{d}^{2} \Phi_{g}$ emitted by the background $m_{2}$ in a direction $\theta_{2}^{\prime}$ such that $n_{0} \sin \theta_{0}^{\prime}=n_{2} \sin \theta_{2}^{\prime}$. When crossing the bounded layer $M_{1}$, the flux is attenuated by a factor $T_{210}\left(\theta_{2}^{\prime}\right)$, equal to $T_{012}\left(\theta_{0}^{\prime}\right)$ according to relation (44). Therefore,

$$
\mathrm{d}^{2} \Phi=T_{012}\left(\theta_{0}^{\prime}\right) \mathrm{d}^{2} \Phi_{g} .
$$

Because of the refraction, the solid angle $d \Omega_{0}$ containing the flux $\mathrm{d}^{2} \Phi$ and the solid angle $\mathrm{d} \Omega_{2}$ containing the flux $\mathrm{d}^{2} \Phi_{g}$ are different and related according to Eq. (10):

$$
\mathrm{d} \Omega_{2}=\left(\frac{n_{0}}{n_{2}}\right)^{2} \frac{\cos \theta_{0}^{\prime}}{\cos \theta_{2}^{\prime}} \mathrm{d} \Omega_{0} .
$$

The flux $\mathrm{d}^{2} \Phi_{g}$ emitted by the background in the direction $\theta_{2}^{\prime}$ within the solid angle $\mathrm{d} \Omega_{2}$, relative to the surface element $\mathrm{d} s$, defines a radiance. Since the background is a
Lambertian emitter of irradiance $W_{g}$, the radiance emitted into any direction is equal to $W_{g} / \pi$. Therefore,

$$
\frac{W_{g}}{\pi}=\frac{\mathrm{d}^{2} \Phi_{g}}{\mathrm{~d} s \cos \theta_{2}^{\prime} \mathrm{d} \Omega_{2}} .
$$

Let us express the reflected radiance $L_{r}\left(\theta_{0}^{\prime}\right)$ using Eqs. (67) and (70)-(73). First, in Eq. (71), we replace $\mathrm{d}^{2} \Phi_{g}$ by $\left(W_{g} / \pi\right) \mathrm{d} s \cos \theta_{2}^{\prime} \mathrm{d} \Omega_{2}$ according to Eq. (73). The resulting expression of $\mathrm{d}^{2} \Phi$ is inserted into Eq. (70), which becomes

$$
L_{r}\left(\theta_{0}^{\prime}\right)=\frac{W_{g}}{\pi} T_{012}\left(\theta_{0}^{\prime}\right) \frac{\cos \theta_{2}^{\prime} \mathrm{d} \Omega_{2}}{\cos \theta_{0}^{\prime} \mathrm{d} \Omega_{0}} .
$$

In Eq. (74) we replace $W_{g}$ with its expression (67) and replace the fraction on the right by $\left(n_{0} / n_{2}\right)^{2}$ according to Eq. (72). Equation (74) becomes

$$
L_{r}\left(\theta_{0}^{\prime}\right)=\frac{1}{\pi}\left(n_{0} / n_{2}\right)^{2} T_{012}\left(\theta_{0}\right) T_{012}\left(\theta_{0}^{\prime}\right) \frac{\rho_{g}}{1-\rho_{g} r_{210}} W_{i} .
$$

The ratio of the reflected radiance to the incident irradiance gives, by definition, the bidirectional reflectance distribution function (BRDF) of the background coated with a nonscattering coloring layer:

$$
\operatorname{BRDF}\left(\theta_{0}, \theta_{0}^{\prime}\right)=\frac{L_{r}\left(\theta_{0}^{\prime}\right)}{W_{i}}=\frac{1}{\pi}\left(n_{0} / n_{2}\right)^{2} T_{012}\left(\theta_{0}^{\prime}\right) \frac{\rho_{g}}{1-\rho_{g} r_{210}} .
$$

By measuring with the same illuminating and measuring conditions the radiance $L_{r e f}=W_{i} / \pi$ reflected by a white reference support, we obtain the reflectance factor $R\left(\theta_{0}, \theta_{0}^{\prime}\right)$ of the background coated with the coloring layer, expressed as the ratio $L_{r}\left(\theta_{0}^{\prime}\right) / L_{r e f}$ :

$$
R\left(\theta_{0}, \theta_{0}^{\prime}\right)=\left(n_{0} / n_{2}\right)^{2} T_{012}\left(\theta_{0}^{\prime}\right) \frac{\rho_{g}}{1-\rho_{g} r_{210}} .
$$

The special case of computing the reflectance of a background coated with one scattering layer is identical to the problem of computing the exact reflectance of a varnished painting. One may verify that expression (77) is identical to the reflectance expression developed by Elias and Simonot [Ref. 9, p. 21, Eq. (2), but with different notation].

Note that in the expressions (69) and (77), the specular surface reflection component $R_{012}\left(\theta_{0}\right)$ has been discarded, as is the case in many photospectrometers.

\section{Background Coated with Multiple Nonscattering Layers}

The expression of the reflectance of a background coated with one nonscattering coloring layer $m_{1}$, Eq. (69), contains three terms relative to the bounded coloring layer: the transmittance $T_{012}\left(\theta_{0}\right)$ for a collimated illumination from medium $m_{0}$, the diffuse reflectance $r_{210}$, and the transmittance $t_{210}$ for a diffuse illumination from medium $m_{2}$ [Eqs. (39), (47), and (49)]. We may extend Eq. (69) directly to the case of $k$ superposed layers $m_{1 \ldots k}$ by considering the superposed layers as a single bounded layer of transmittance $T_{0 \ldots k+1}\left(\theta_{0}\right)$ for a collimated illumination from medium $m_{0}$ [Eqs. (58)], of diffuse reflectance $r_{k+1 \ldots 0}$ and diffuse transmittance $t_{k+1 \ldots 0}$ for a diffuse illumina- 
tion from medium $m_{k+1}$ [Eqs. (62) and (63) with exchanged subscripts 0 and $k+1$ ]. The reflectance $R_{m 1 \ldots k}$ of the background coated with $k$ superposed layers $m_{1 \ldots k}$ is therefore

$$
R_{m 1 \ldots k}=t_{k+1 \ldots 0} \frac{\rho_{g} T_{0 \ldots k+1}\left(\theta_{0}\right)}{1-\rho_{g} r_{k+1 \ldots 0}} .
$$

In analogy to expression (77), for a collimated illumination with incidence $\theta_{0}$, for an observation angle $\theta_{0}^{\prime}$, and for a perfectly white diffuse reflector as reference, the reflectance factor of the background coated with $k$ superposed layers is

$$
R\left(\theta_{0}, \theta_{0}^{\prime}\right)=\left(n_{0} / n_{2}\right)^{2} T_{0 \ldots k+1}\left(\theta_{0}\right) T_{0 \ldots k+1}\left(\theta_{0}^{\prime}\right) \frac{\rho_{g}}{1-\rho_{g} r_{k+1 \ldots 0}} .
$$

\section{Particular Case: The Williams-Clapper Model}

Because of the multiple internal reflections taking place at both sides of the interface in contact with the diffusing background, the reflectance model presented here is slightly different from the Williams-Clapper model. ${ }^{2}$ In the Williams-Clapper model, the nonscattering layer $m_{1}$ and the diffusing background $m_{2}$ are assumed to have the same refractive index, i.e., $n_{1}=n_{2}$. Therefore, at the interface $i_{12}$, the Fresnel coefficients are $R_{21}(\theta)=R_{12}(\theta)=0$ (no reflection) and $T_{21}(\theta)=T_{12}(\theta)=1$ (total transmission). According to Eqs. (30), (33), (37), and (39)-(41), $R_{012}(\theta)$ reduces to $R_{01}(\theta), R_{210}(\theta)$ becomes $R_{10}(\theta) t_{1}^{2}(\theta)$, and $T_{012}(\theta)$ becomes $T_{01}(\theta) t_{1}(\theta)$, with $t_{1}(\theta)$ given by Eq. (23). The term $r_{210}$ becomes

$$
r_{110}=\int_{\theta=0}^{\pi / 2} R_{10}(\theta) t_{1}^{2}(\theta) \sin 2 \theta \mathrm{d} \theta,
$$

and the term $t_{210}$ becomes

$$
t_{110}=\int_{\theta=0}^{\pi / 2} T_{10}(\theta) t_{1}\left(\theta_{1}\right) \sin 2 \theta \mathrm{d} \theta .
$$

By inserting these simplified expressions into Eq. (77), we retrieve the reflectance factor of the Williams-Clapper model, expressed for a collimated incidence and observation (respective angles $\theta_{0}$ and $\theta_{0}^{\prime}$ ):

$$
R\left(\theta_{0}, \theta_{0}^{\prime}\right)=\left(n_{0} / n_{1}\right)^{2} \frac{\rho_{g} T_{01}\left(\theta_{0}\right) T_{01}\left(\theta_{0}^{\prime}\right) t_{1}\left(\theta_{1}\right) t_{1}\left(\theta_{1}^{\prime}\right)}{1-\rho_{g} r_{110}} .
$$

In the case of a collimated illumination and an integrating sphere measuring geometry, the reflectance given by Eq. (69) reduces to

$$
R_{m 1}=t_{110} \frac{\rho_{g} T_{01}\left(\theta_{0}\right) t_{1}\left(\theta_{1}\right)}{1-\rho_{g} r_{110}}
$$

We retrieve with this expression (83) the ShoreSpoonhower generalization ${ }^{6}$ of the Williams-Clapper model for an integrating-sphere measuring geometry, also derived independently by Elias et al. ${ }^{7}$

\section{E. Intrinsic Reflectance of the Background}

The diffusing background is characterized by its intrinsic reflectance $\rho_{g}$. However $\rho_{g}$ is not directly measurable, since the interface that separates the background (medium 2) and the air (medium 0) induces multiple internal reflections. However, it is possible to measure the reflectance of the background and derive its intrinsic reflectance $\rho_{g}$ from Eq. (69) if the measurements are performed with an integrating sphere or from Eq. (77) if they are performed with a radiance detector.

The background, medium $m_{2}$, is directly in contact with the medium $m_{0}$. Thus, the absorption coefficient is $\alpha=0$; the terms $T_{012}\left(\theta_{0}\right)$, and $T_{012}\left(\theta_{0}^{\prime}\right)$ become, respectively, $T_{02}\left(\theta_{0}\right)$ and $T_{02}\left(\theta_{0}^{\prime}\right)$; and the terms $r_{210}$ and $t_{210}$ become, respectively, $r_{20}$ and $t_{20}$. According to Eq. (69), the reflectance $\rho$ of the background measured with an integrating sphere is

$$
\rho=t_{20} \frac{\rho_{g} T_{02}\left(\theta_{0}\right)}{1-\rho_{g} r_{20}}
$$

and according to Eq. (77), the reflectance factor of the background measured with a radiance detector is

$$
R\left(\theta_{0}, \theta_{0}^{\prime}\right)=\left(n_{0} / n_{2}\right)^{2} T_{02}\left(\theta_{0}\right) T_{02}\left(\theta_{0}^{\prime}\right) \frac{\rho_{g}}{1-\rho_{g} r_{20}}
$$

The intrinsic reflectance $\rho_{g}$ of the background can be obtained by inversion of Eq. (84)

$$
\rho_{g}=\frac{\rho}{t_{20} T_{02}\left(\theta_{0}\right)+r_{20} \rho}
$$

or, respectively, by inversion of Eq. (85):

$$
\rho_{g}=\frac{R\left(\theta_{0}, \theta_{0}^{\prime}\right)}{\left(n_{0} / n_{2}\right)^{2} T_{02}\left(\theta_{0}\right) T_{02}\left(\theta_{0}^{\prime}\right)+r_{20} R\left(\theta_{0}, \theta_{0}^{\prime}\right)} .
$$

\section{CONCLUSIONS}

We propose a model for predicting the reflectance and transmittance of multiple stacked nonscattering coloring layers that have distinct refractive indices. The model relies on the modeling of the reflectance and transmittance of a bounded coloring layer, i.e., a coloring layer and its two interfaces with neighboring media of different refractive indices. By replacing within the expressions for the bounded layer reflectance (respectively, transmittance), the reflectance (respectively, transmittance) of a simple interface with the reflectance (respectively, transmittance) of a bounded layer, we are able to deduce the reflectance and transmittance of multiple stacked nonscattering layers of different refractive indices. This layer composition rule is then applied to deduce the reflectance of stacked nonscattering layers of distinct refractive indices superposed with a reflecting diffusing background that has its own refractive index. The Williams-Clapper model $^{2}$ as well as the air-paint ${ }^{7}$ and the air-varnish-paint ${ }^{9}$ reflection models become special cases of the proposed stacked layer model. Since the proposed 
model takes into account different illumination and measuring conditions, it is well suited for practical applications.

The corresponding author's e-mail address is lionel.simonot@univ-poitiers.fr.

\section{REFERENCES}

1. P. Kubelka and F. Munk, "Ein Beitrag zur Optik der Farbanstriche," Z. Tech. Phys. 12, 593-601 (1931).

2. F. C. Williams and F. R. Clapper, "Multiple internal reflections in photographic color prints," J. Opt. Soc. Am. 43, 595-599 (1953).

3. P. Kubelka, "New contributions to the optics of intensely light-scattering material. Part I,” J. Opt. Soc. Am. 38, 448-457 (1948).

4. P. Kubelka, "New contributions to the optics of intensely light-scattering materials. Part II: Nonhomogenous layers," J. Opt. Soc. Am. 44, 330-335 (1954).
5. J. L. Saunderson, "Calculation of the color pigmented plastics,” J. Opt. Soc. Am. 32, 727-736 (1942).

6. J. D. Shore and J. P. Spoonhower, "Reflection density in photographic color prints: generalizations of the Williams-Clapper transform,” J. Imaging Sci. Technol. 45, 484-488 (2001).

7. M. Elias, L. Simonot, and M. Menu, "Bidirectional reflectance of a diffuse background covered by a partly absorbing layer," Opt. Commun. 191, 1-7 (2001).

8. M. Hebert and R. D. Hersch, "Classical print reflection models: a radiometric approach," J. Imaging Sci. Technol. 48, 363-374 (2004).

9. M. Elias and L. Simonot, "Bi-directional reflectance of a varnished painting part 1: influence of the refractive indices without using the approximations of Saunderson correction-exact computation," Opt. Commun. 231, 17-24 (2004).

10. M. Born and E. Wolfe, Principles of Optics, 6th ed. (Pergamon, 1987), Sec. 1.5.

11. W. R. McCluney, Introduction to Radiometry and Photometry (Artech House, 1994), pp. 7-13.

12. D. B. Judd, "Fresnel reflection of diffusely incident light," J. Res. Natl. Bur. Stand. 29, 329-332 (1942). 